\title{
VOLUME BOUNDS OF THE RICCI FLOW ON CLOSED MANIFOLDS
}

\author{
CHIH-WEI CHEN AND ZHENLEI ZHANG
}

\begin{abstract}
Let $\{g(t)\}_{t \in[0, T)}$ be the solution of the Ricci flow on a closed Riemannian manifold $M^{n}$ with $n \geq 3$. Without any assumption, we derive lower volume bounds of the form $\operatorname{Vol}_{g(t)} \geq C(T-t)^{\frac{n}{2}}$, where $C$ depends only on $n, T$ and $g(0)$. In particular, we show that

$$
\operatorname{Vol}_{g(t)} \geq e^{T \lambda-\frac{n}{2}}\left(\frac{4}{(A(\lambda-r)+4 B) T}\right)^{\frac{n}{2}}(T-t)^{\frac{n}{2}}
$$

where $r:=\inf _{\|\phi\|_{2}^{2}=1} \int_{M} R \phi^{2} d \operatorname{vol}_{g(0)}, \lambda:=\inf _{\|\phi\|_{2}^{2}=1} \int_{M} 4|\nabla \phi|^{2}+R \phi^{2} d \operatorname{vol}_{g(0)}$ and $A, B$ are Sobolev constants of $(M, g(0))$. This estimate is sharp in the sense that it is achieved by the unit sphere with scalar curvature $R_{g(0)}=n(n-1)$ and $A=$ $\frac{4}{n(n-2)} \omega_{n}^{-\frac{2}{n}}, B=\frac{n-1}{n-2} \omega_{n}^{-\frac{2}{n}}$.

On the other hand, if the diameter satisfies $\operatorname{diam}_{g(t)} \leq c_{1} \sqrt{T-t}$ and there exist a point $x_{0} \in M$ such that $R\left(x_{0}, t\right) \leq c_{2}(T-t)^{-1}$, then we have $\operatorname{Vol}_{g(t)} \leq C(T-t)^{\frac{n}{2}}$ for all $t>\frac{T}{2}$, where $C$ depends only on $c_{1}, c_{2}, n, T$ and $g(0)$.
\end{abstract}

\section{INTRODUCTION}

Let $\left(M^{n}, g\right)$ be a closed Riemannian manifold with dimension $n \geq 3$ and $A$ and $B$ be any Sobolev constants of $\left(M^{n}, g\right)$, i.e.,

$$
\left(\int_{M}|u|^{\frac{2 n}{n-2}} d \mathrm{vol}\right)^{\frac{n-2}{n}} \leq A \int_{M}|\nabla u|^{2} d \mathrm{vol}+B \int_{M} u^{2} d \mathrm{vol}
$$

for all $u \in W^{1,2}(M)$. In [ZZha07], one of us observed that the Ricci flow on a closed manifold has a volume lower bound in terms of Sobolev constants. Especially, when $\lambda:=\inf _{\|\phi\|_{2}^{2}=1} \int_{M} 4|\nabla \phi|^{2}+R \phi^{2} d \operatorname{vol}_{g(0)}$ is non-positive, one obtains $\operatorname{Vol}_{g(t)} \geq C_{1} e^{-C_{2} t}$ for all $t>0$, where $C_{1}$ and $C_{2}$ depend only on $n$ and $g(0)$. It means that the manifold cannot extinct at finite time and every blow up limit must be non-compact.

On the other hand, for positive $\lambda$, R. Ye [Ye07] derived the following volume lower bound by using the estimate of $A(t)$ and $B(t)$ :

Proposition 1 (R. Ye). Assume that $\lambda$ is positive. Then we have for any time $t \in$ $[0, T)$

$$
\operatorname{Vol}_{g(t)} \geq e^{-\frac{1}{4}-C} \text { when } \bar{R}(t) \leq 0
$$

and

$$
\operatorname{Vol}_{g(t)} \geq e^{-\frac{1}{4}-C} \bar{R}(t)^{-\frac{n}{2}} \text { when } \bar{R}(t)>0,
$$

where $\bar{R}(t):=f R d \operatorname{vol}_{g(t)}$ and $C$ depends on $n, g_{0}, A, B, \lambda, \operatorname{Vol}_{g(0)}$ and $\max R_{g(0)}^{-}$.

Date: March, 2018.

Key words and phrases. Ricci flow, volume estimate, $\mu$-entropy. 
Note that the constants $C$ 's in Zhang's estimate depend only on the initial metric $g(0)$, while the constants in Ye's estimate, namely for the case $\lambda>0$, might depend on $\bar{R}(t)$.

In this article, we find a unified way to derive several volume bounds, whose proofs do not rely on the definite sign of $\lambda$.

Theorem 1. Let $\{g(t)\}_{t \in[0, T)}$ be the solution of the Ricci flow on a closed Riemannian manifold $M^{n}$ with $n \geq 3$ and $A, B$ be Sobolev constants of $(M, g(0))$. Then

$$
\operatorname{Vol}_{g(t)} \geq e^{T \lambda-\frac{n a}{8}(A(\lambda-r)+4 B)} a^{\frac{n}{2}}\left(\frac{T-t}{T}\right)^{\frac{n}{2}}
$$

for all $a \in\left(0, \frac{8 T}{n A}\right]$, where $r:=\inf _{\|\phi\|_{2}^{2}=1} \int_{M} R \phi^{2} d \operatorname{vol}_{g(0)}$ and $\lambda:=\inf _{\|\phi\|_{2}^{2}=1} \int_{M} 4|\nabla \phi|^{2}+$ $R \phi^{2} d \operatorname{vol}_{g(0)}$. In particular, when $a=\frac{8 T}{n A}$, we obtain a lower bound

$$
\operatorname{Vol}_{g(t)} \geq e^{T\left(r-4 B A^{-1}\right)}\left(\frac{8}{n A}\right)^{\frac{n}{2}}(T-t)^{\frac{n}{2}}
$$

which does not depend on $\lambda$.

When choosing $B \geq \frac{n A}{8 T}$ and $a=4(A(\lambda-r)+4 B)^{-1}$, we have the following theorem which shows that our estimate is sharp.

Theorem 2. Let $\{g(t)\}_{t \in[0, T)}$ be the solution of the Ricci flow on a closed Riemannian manifold $M^{n}$ with $n \geq 3$. Suppose that $A$ and $B \geq \frac{n A}{8 T}$ are Sobolev constants of $(M, g(0))$. Then

$$
\operatorname{Vol}_{g(t)} \geq e^{T \lambda-\frac{n}{2}}\left(\frac{4}{A(\lambda-r)+4 B}\right)^{\frac{n}{2}}\left(\frac{T-t}{T}\right)^{\frac{n}{2}} .
$$

The bound is achieved when $(M, g(0))$ is the unit sphere with $A=\frac{4}{n(n-2)} \omega_{n}^{-\frac{2}{n}}$ and $B=\frac{n-1}{n-2} \omega_{n}^{-\frac{2}{n}}$.

The proof of Theorem 1 is based on the monotonicity of Perelman's $\mu$-entropy. Recall that $\mu$-entropy is defined by

$$
\mu(g(t), \tau(t)):=\inf _{\|\phi\|_{2}^{2}=(4 \pi \tau)^{\frac{n}{2}}}(4 \pi \tau)^{-\frac{n}{2}} \int_{M}\left[\tau\left(4|\nabla \phi|^{2}+R \phi^{2}\right)-\phi^{2} \ln \phi^{2}-n \phi^{2}\right] d \operatorname{vol}_{g(t)}
$$

for all $\phi(\cdot, t) \in W^{1,2}(M)$. When fixing $t$ and choosing $\phi^{2}$ to be the constant $(4 \pi \tau)^{\frac{n}{2}} \operatorname{Vol}_{g(t)}^{-1}$, one obtains

$$
\mu(g(t), \tau(t)) \leq f_{M} \tau R d \operatorname{vol}_{g}-\ln \frac{(4 \pi \tau)^{\frac{n}{2}}}{\operatorname{Vol}_{g(t)}}-n=-\tau\left(\ln \operatorname{Vol}_{g(t)}\right)^{\prime}-\ln \frac{(4 \pi \tau)^{\frac{n}{2}}}{\operatorname{Vol}_{g(t)}}-n
$$

This shows that the evolution of volume is closely related to the $\mu$-entropy. The relationship between $\mu$ and Vol has been studied by one of the authors in [ZZha07, especially for manifolds with $\lambda<0$. Here we derive results for generic manifolds.

Theorem 3. Let $\{g(t)\}_{t \in[0, T)}$ be the solution of the Ricci flow on a closed Riemannian manifold $M^{n}$ with $n \geq 2$. Denote $\mu=\inf _{\|\phi\|_{2}^{2}=(4 \pi T)^{\frac{n}{2}}} \mathcal{W}(g(0), \phi, T)$. Then

$$
\operatorname{Vol}_{g(t)} \geq(4 \pi)^{\frac{n}{2}} e^{\mu+\frac{n}{2}}(T-t)^{\frac{n}{2}} .
$$


As a consequence of the volume lower bound, for any closed Riemannian manifold $(M, g)$, one has

$$
\mu(g, T) \leq-\frac{n}{2}+\ln \operatorname{Vol}_{g}(M)-\frac{n}{2} \ln 4 \pi T .
$$

In particular, for any Ricci flow defined on a closed manifold, the maximal time $T$ cannot exceed $(4 \pi e)^{-1}\left(e^{-\mu(g(0), T)} \operatorname{Vol}_{g(0)}\right)^{\frac{2}{n}}$.

If we further assume some controls on diameter and curvature, we derive the following upper bound for volume.

Proposition 2. Let $\{g(t)\}_{t \in[0, T)}$ be the solution of the Ricci flow on a closed Riemannian manifold $\left(M^{n}, g(0)\right)$ with $n \geq 3$. If the diameter satisfies $\operatorname{diam}_{g(t)} \leq c_{1} \sqrt{T-t}$ and there exist a point $x_{0} \in M$ such that $R\left(x_{0}, t\right) \leq c_{2}(T-t)^{-1}$, then we have $\mathrm{Vol}_{g(t)} \leq C(T-t)^{\frac{n}{2}}$ for all $t>\frac{T}{2}$, where $C$ depends only on $c_{1}, c_{2}, n, T$ and $g(0)$.

If the curvature condition in Proposition 2 is replaced by the stronger one that $R(x, t) \leq c_{2}(T-t)^{-1}$ for all $x \in M$, then the theorem follows directly from Q. Zhang's Theorem 1.1 in QZha12. However, by mimicking Zhang's argument carefully, one can see that the Type I curvature assumption can be reduced as $R\left(x_{0}, t\right) \leq c_{1}(T-t)^{-1}$ for some $x_{0} \in M$. For the reader's convenience, we include an outline of Zhang's proof in Section 6.

The theorem above relates to the following conjecture. Roughly speaking, we suspect that $R$ cannot be of Type II at every point on a manifold which shrinks to a point along the Ricci flow.

Conjecture. Let $\{g(t)\}_{t \in[0, T)}$ be the solution of the Ricci flow on a closed Riemannian manifold $M^{n}$ with $n \geq 3$ and $\operatorname{diam}_{g(t)} \rightarrow 0$ as $t \rightarrow T$. Then

$$
\liminf _{t \rightarrow T}(T-t) R(x, t)<\infty
$$

Acknowledgement. The first author appreciates Mao-Pei Tsui for suggesting him to compare the volume of sphere and other manifolds. He is always indebted to Shu-Cheng Chang and Huai-Dong Cao for their constant supports and discussions.

\section{LOWER BOUndS INVOLVING SobOLEV CONSTANTS}

Let $\{g(x, t)\}_{t \in[0, T)}$ be the solution of the Ricci flow on a closed Riemannian manifold $M^{n}$ with $n \geq 3$. Perelman's $\mathcal{W}$-functional is defined by

$$
\mathcal{W}(g(x, t), \phi(x, t), \tau(t)):=(4 \pi \tau)^{-\frac{n}{2}} \int_{M}\left[\tau\left(4|\nabla \phi|^{2}+R \phi^{2}\right)-\phi^{2} \ln \phi^{2}-n \phi^{2}\right] d \operatorname{vol}_{g}
$$

for all $\phi(\cdot, t) \in W^{1,2}(M)$.

Perelman proved the monotonicity of $\mathcal{W}$ along the Ricci flow Per02. Precisely, for any $\tau(t)$ and $\phi$ such that $\tau^{\prime}=-1$ and $\frac{\partial}{\partial t} \phi^{2}=-\Delta \phi^{2}+\left(R-\frac{n}{2 \tau}\right) \phi^{2}, \mathcal{W}(g(x, t), \phi(x, t), \tau(t))$ and $\mu:=\inf _{\|\phi\|_{2}^{2}=(4 \pi \tau)^{\frac{n}{2}}} \mathcal{W}(g(x, t), \phi(x, t), \tau(t))$ are nondecreasing with respect to $t$. In particular, the value of $\mu$ at $t>0$ is greater than or equal to the value at $t=0$. Namely,

$$
\inf _{\|\phi\|_{2}^{2}=(4 \pi \tau(t))^{\frac{n}{2}}} \mathcal{W}(g(t), \phi, \tau(t)) \geq \inf _{\|\phi\|_{2}^{2}=(4 \pi \tau(0))^{\frac{n}{2}}} \mathcal{W}(g(0), \phi, \tau(0))
$$


and equivalently

$$
\sup _{\|\phi\|_{2}^{2}=(4 \pi \tau(t))^{\frac{n}{2}}}-\mathcal{W}(g(t), \phi, \tau(t)) \leq \sup _{\|\phi\|_{2}^{2}=(4 \pi \tau(0))^{\frac{n}{2}}}-\mathcal{W}(g(0), \phi, \tau(0)) .
$$

Hence

$$
\begin{aligned}
& \sup _{\|\phi\|_{2}^{2}=(4 \pi \tau(t))^{\frac{n}{2}}}(4 \pi \tau(t))^{-\frac{n}{2}} \int_{M}\left[\phi^{2} \ln \phi^{2}+n \phi^{2}-\tau(t)\left(4|\nabla \phi|^{2}+R \phi^{2}\right)\right] d \operatorname{vol}_{g(t)} \\
\leq & \sup _{\|\phi\|_{2}^{2}=(4 \pi \tau(0))^{\frac{n}{2}}}(4 \pi \tau(0))^{-\frac{n}{2}} \int_{M}\left[\phi^{2} \ln \phi^{2}+n \phi^{2}-\tau(0)\left(4|\nabla \phi|^{2}+R \phi^{2}\right)\right] d \operatorname{vol}_{g(0)} .
\end{aligned}
$$

From now on, we denote $\tau=\tau(t), \tau_{0}=\tau(0)$ and $V(t)=\operatorname{Vol}_{g(t)}$. Considering the (spatial) constant function $\phi^{2}=(4 \pi \tau)^{\frac{n}{2}} V(t)^{-1}$ at time $t$, one derives

$$
\begin{aligned}
& f_{M}\left[\ln \frac{(4 \pi \tau)^{\frac{n}{2}}}{V(t)}+n-\tau R\right] d \operatorname{vol}_{g(t)} \\
\leq & \sup _{\|\phi\|_{2}^{2}=\left(4 \pi \tau_{0}\right)^{\frac{n}{2}}}\left(4 \pi \tau_{0}\right)^{-\frac{n}{2}} \int_{M}\left[\phi^{2} \ln \phi^{2}+n \phi^{2}-\tau_{0}\left(4|\nabla \phi|^{2}+R \phi^{2}\right)\right] d \operatorname{vol}_{g(0)} \\
= & \sup _{\|\phi\|_{2}^{2}=1} \int_{M}\left[\phi^{2}\left(\ln \phi^{2}+\ln \left(4 \pi \tau_{0}\right)^{\frac{n}{2}}\right)+n \phi^{2}-\tau_{0}\left(4|\nabla \phi|^{2}+R \phi^{2}\right)\right] d \operatorname{vol}_{g(0)},
\end{aligned}
$$

i.e.,

$$
\begin{aligned}
& -\ln V(t)+\ln (4 \pi \tau)^{\frac{n}{2}}-\tau f_{M} R d \operatorname{vol}_{g(t)} \\
\leq & \sup _{\|\phi\|_{2}^{2}=1} \int_{M}\left[\phi^{2} \ln \phi^{2}-\tau_{0}\left(4|\nabla \phi|^{2}+R \phi^{2}\right)\right] d \operatorname{vol}_{g(0)}+\ln \left(4 \pi \tau_{0}\right)^{\frac{n}{2}} .
\end{aligned}
$$

On the other hand, since $\tau^{\prime}=-1$ and $\frac{d}{d t} V(t)=-\int_{M} R d$ vol, one has

$$
\frac{d}{d t}(\tau \ln V(t))=-\ln V(t)-\tau f_{M} R d \mathrm{vol}
$$

Therefore,

$$
\frac{d}{d t}(\tau \ln V(t)) \leq \sup _{\|\phi\|_{2}^{2}=1} \int_{M}\left[\phi^{2} \ln \phi^{2}-\tau_{0}\left(4|\nabla \phi|^{2}+R \phi^{2}\right)\right] d \operatorname{vol}_{g(0)}-\ln \left(\frac{\tau}{\tau_{0}}\right)^{\frac{n}{2}} .
$$

Inequalities (11) and the right hand side of (2) were observed and used before, see for example [QZha07, Ye07]. The left hand side of (2) also occurred in a more general form in QZha07, QZha12. However, they were used for tracing the evolution of Sobolev constants or fundamental solutions of the (conjugate) heat equation, instead of the global volume function $V(t)$. In Ye's Proposition (cf. Proposition 1 in the introduction), Ye needs the positivity assumption to make sure that $A(t)$ and $B(t)$ are under control along the Ricci flow. Indeed, as pointed out by Ye, when the assumption $\lambda>0$ is removed, Hamilton-Isenberg's example shows that local volume could collapse, which means that $A(t)$ and $B(t)$ must become wild. So Ye guessed that the positivity assumption of $\lambda$ is indispensable [Ye07, p. 4]. However, we show that, although the Sobolev constants and the local volume could be bad, the global volume remains under control. 
Theorem 1. Let $\{g(t)\}_{t \in[0, T)}$ be the solution of the Ricci flow on a closed Riemannian manifold $M^{n}$ with $n \geq 3$ and $A, B$ be Sobolev constants of $(M, g(0))$. Then

$$
\operatorname{Vol}_{g(t)} \geq e^{T \lambda-\frac{n a}{8}(A(\lambda-r)+4 B)} a^{\frac{n}{2}}\left(\frac{T-t}{T}\right)^{\frac{n}{2}}
$$

for all $a \in\left(0, \frac{8 T}{n A}\right]$, where $r:=\inf _{\|\phi\|_{2}^{2}=1} \int_{M} R \phi^{2} d \operatorname{vol}_{g(0)}$ and $\lambda:=\inf _{\|\phi\|_{2}^{2}=1} \int_{M} 4|\nabla \phi|^{2}+$ $R \phi^{2} d \operatorname{vol}_{g(0)}$. In particular, when $a=\frac{8 T}{n A}$, we obtain a lower bound

$$
\operatorname{Vol}_{g(t)} \geq e^{T\left(r-4 B A^{-1}\right)}\left(\frac{8}{n A}\right)^{\frac{n}{2}}(T-t)^{\frac{n}{2}},
$$

which does not depend on $\lambda$.

Proof. For all $\phi \in W^{1,2}(M)$, the Sobolev inequality implies that

$$
\begin{aligned}
\int_{M} \phi^{2} \ln \phi^{2} d \operatorname{vol}_{g(0)} & \leq \frac{n}{2} \ln \left(A \int_{M}|\nabla \phi|^{2} d \operatorname{vol}_{g(0)}+B \int_{M} \phi^{2} d \operatorname{vol}_{g(0)}\right) \\
& \leq \frac{n}{2} a\left(A \int_{M}|\nabla \phi|^{2} d \operatorname{vol}_{g(0)}+B \int_{M} \phi^{2} d \operatorname{vol}_{g(0)}\right)-\frac{n}{2} \ln a-\frac{n}{2} \\
& =\frac{n a A}{8} \int_{M} 4|\nabla \phi|^{2} d \operatorname{vol}_{g(0)}+\frac{n a B}{2} \int_{M} \phi^{2} d \operatorname{vol}_{g(0)}-\frac{n}{2} \ln a-\frac{n}{2}
\end{aligned}
$$

Note that the second inequality follows from the fact $\ln x \leq a x-\ln a-1$. Hence

$$
\begin{aligned}
& \int_{M}\left[\phi^{2} \ln \phi^{2}-\tau_{0}\left(4|\nabla \phi|^{2}+R \phi^{2}\right)\right] d \operatorname{vol}_{g(0)} \\
\leq & \left(\frac{n a A}{8}-\tau_{0}\right) \int_{M} 4|\nabla \phi|^{2}+R \phi^{2} d \operatorname{vol}_{g(0)}-\frac{n a A}{8} \int_{M} R \phi^{2} d \operatorname{vol}_{g(0)} \\
& +\frac{n a B}{2} \int_{M} \phi^{2} d \operatorname{vol}_{g(0)}-\frac{n}{2} \ln a-\frac{n}{2} .
\end{aligned}
$$

Since $a \leq \frac{8 \tau_{0}}{n A}, \frac{n a A}{8}-\tau_{0}$ is nonpositive and we have

$$
\begin{aligned}
& \sup _{\|\phi\|_{2}^{2}=1} \int_{M}\left[\phi^{2} \ln \phi^{2}-\tau_{0}\left(4|\nabla \phi|^{2}+R \phi^{2}\right)\right] d \operatorname{vol}_{g(0)} \\
\leq & \left(\frac{n a A}{8}-\tau_{0}\right) \lambda-\frac{n a A}{8} r+\frac{n a B}{2}-\frac{n}{2} \ln a-\frac{n}{2} \\
= & -\tau_{0} \lambda+\frac{n a A}{8}(\lambda-r)+\frac{n a B}{2}-\frac{n}{2} \ln a-\frac{n}{2},
\end{aligned}
$$

where $\lambda:=\inf _{\|\phi\|_{2}^{2}=1} \int_{M} 4|\nabla \phi|^{2}+R \phi^{2} d \operatorname{vol}_{g(0)}$ and $r:=\inf _{\|\phi\|_{2}^{2}=1} \int_{M} R \phi^{2} d \operatorname{vol}_{g(0)}$.

Applying it to the key inequality (2), we obtain

$$
\begin{aligned}
\frac{d}{d t}(\tau \ln V(t)) & \leq \sup _{\|\phi\|_{2}^{2}=1} \int_{M}\left[\phi^{2} \ln \phi^{2}-\tau_{0}\left(4|\nabla \phi|^{2}+R \phi^{2}\right)\right] d \operatorname{vol}_{g(0)}-\ln \left(\frac{\tau}{\tau_{0}}\right)^{\frac{n}{2}} \\
& \leq-\tau_{0} \lambda+\frac{n a A}{8}(\lambda-r)+\frac{n a B}{2}-\frac{n}{2} \ln a-\frac{n}{2}-\ln \left(\frac{\tau}{\tau_{0}}\right)^{\frac{n}{2}}
\end{aligned}
$$

Taking $\tau=T-t$ and integrating the inequality from $t$ to $T$, we have

$$
-\ln V(t) \leq-T \lambda+\frac{n a A}{8}(\lambda-r)+\frac{n a B}{2}-\ln \left(\frac{a}{T}\right)^{\frac{n}{2}}-\ln (T-t)^{\frac{n}{2}},
$$


i.e.,

$$
V(t) \geq e^{T \lambda-\frac{n a A}{8}(\lambda-r)-\frac{n a B}{2}} a^{\frac{n}{2}}\left(\frac{T-t}{T}\right)^{\frac{n}{2}}
$$

for all $t \in[0, T)$. It is easy to see that $\lambda$ can be cancelled out when $a=\frac{8 T}{n A}$.

\section{Sharp volume Bound and the Best Sobolev Constants of the SPhere}

In order to acquire a sharp estimate, one has to choose a specific constant $a$ in Theorem 1. In fact, the lower bound in Theorem 1 involves the function $f(a)=e^{-C a} a^{\frac{n}{2}}$, which has a unique absolute maximum at $a=\frac{n}{2 C}$. So it is not hard to see that the best choice of $a$ is $4(A(\lambda-r)+4 B)^{-1}$. However, due to a technical but crucial reason arising from the proof, $a$ cannot exceed $\frac{8 T}{n A}$. Hence the best choice of $a$ is allowed only when $B$ is chosen to be large, say $B \geq \frac{n A}{8 T}$. This fact shows that the best choice of $a$ is not necessarily given by the best Sobolev constants.

The best choice of $a$, where $f(a)$ attains it maximum, makes the lower bound in Theorem 1 becomes $e^{T \lambda-\frac{n}{2}} a^{\frac{n}{2}}\left(\frac{T-t}{T}\right)^{\frac{n}{2}}$. We shall recall some facts from the theory of Sobolev constants (cf. [DH02]) and show that this lower bound can be attained by the shrinking sphere. From now on we consider closed Riemannian manifolds with dimension $n \geq 3$ and denote $\omega_{n}$ as the volume of the unit sphere, whose sectional curvatures are 1. In [HV96], Hebey and Vaugon showed that one can always choose $A=\frac{4}{n(n-2)} \omega_{n}^{-\frac{2}{n}}$ for a given $\left(M^{n}, g\right)$ so that the Sobolev inequality holds. Namely, there exists a constant $B>0$ such that

$$
\left(\int_{M}|u|^{\frac{2 n}{n-2}} d \mathrm{vol}\right)^{\frac{n-2}{n}} \leq \frac{4}{n(n-2)} \omega_{n}^{-\frac{2}{n}} \int_{M}|\nabla u|^{2} d \mathrm{vol}+B \int_{M} u^{2} d \mathrm{vol}
$$

for all $u \in W^{1,2}(M)$. The infimum of all the $B$ 's which make the inequality valid is called the best $B$-constant and is denoted by $B_{0}$. For the unit sphere, a well-known result due to T. Aubin [Aub76] states that $B_{0}$ is $\omega_{n}^{-\frac{2}{n}}$. Hence, the Sobolev inequality holds on the unit sphere when we choose $B=\frac{n-1}{n-2} \omega_{n}^{-\frac{2}{n}}>B_{0}$ and we have

Theorem 2. Let $\{g(t)\}_{t \in[0, T)}$ be the solution of the Ricci flow on a closed Riemannian manifold $M^{n}$ with $n \geq 3$. Suppose that $A$ and $B \geq \frac{n A}{8 T}$ are Sobolev constants of $(M, g(0))$. Then

$$
\operatorname{Vol}_{g(t)} \geq e^{T \lambda-\frac{n}{2}}\left(\frac{4}{A(\lambda-r)+4 B}\right)^{\frac{n}{2}}\left(\frac{T-t}{T}\right)^{\frac{n}{2}}
$$

The bound is achieved when $(M, g(0))$ is the unit sphere with $A=\frac{4}{n(n-2)} \omega_{n}^{-\frac{2}{n}}$ and $B=\frac{n-1}{n-2} \omega_{n}^{-\frac{2}{n}}$.

Proof. The first statement follows easily from Theorem 1 by taking $a=4(A(\lambda-r)+$ $4 B)^{-1}$.

For the second statement, we consider the shrinking sphere with $R_{g(0)}=n(n-1)$, it is easy to compute that $T=\frac{1}{2(n-1)}$ and $\operatorname{Vol}_{g(t)}=(2(n-1))^{\frac{n}{2}} \omega_{n}(T-t)^{\frac{n}{2}}$. On the other 
hand, when we choose $A=\frac{4}{n(n-2)} \omega_{n}^{-\frac{2}{n}}$ and $B=\frac{n-1}{n-2} \omega_{n}^{-\frac{2}{n}}$, the lower bound becomes

$$
e^{\frac{1}{2(n-1)} n(n-1)-\frac{n}{2}}\left(2(n-1) \frac{4}{A(\lambda-r)+4 B}\right)^{\frac{n}{2}}(T-t)^{\frac{n}{2}}=(2(n-1))^{\frac{n}{2}} \omega_{n}(T-t)^{\frac{n}{2}}
$$

because $\lambda=r=R=n(n-1)$.

\section{LOWER BOUNDS INVOLVING $\mu$}

In this section, we derive lower and upper bounds of global volume in terms of $\mu(g(0), T)$. Since we do not interpret $\mu$ by using Sobolev constants, all the results in this section hold for $n \geq 2$, instead of $n \geq 3$.

Theorem 3. Let $\{g(t)\}_{t \in[0, T)}$ be the solution of the Ricci flow on a closed Riemannian manifold $M^{n}$ with $n \geq 2$. Denote $\mu=\inf _{\|\phi\|_{2}^{2}=(4 \pi T)^{\frac{n}{2}}} \mathcal{W}(g(0), \phi, T)$. Then

$$
\operatorname{Vol}_{g(t)} \geq(4 \pi)^{\frac{n}{2}} e^{\mu+\frac{n}{2}}(T-t)^{\frac{n}{2}} .
$$

As a consequence of the volume lower bound, for any closed Riemannian manifold $(M, g)$, one has

$$
\mu(g, T) \leq-\frac{n}{2}+\ln \operatorname{Vol}_{g}(M)-\frac{n}{2} \ln 4 \pi T .
$$

In particular, for any Ricci flow defined on a closed manifold, the maximal time $T$ cannot exceed $(4 \pi e)^{-1}\left(e^{-\mu(g(0), T)} \operatorname{Vol}_{g(0)}\right)^{\frac{2}{n}}$.

Proof. Recall that Perelman's $\mu$-entropy

$$
\begin{aligned}
\mu(g(t), \tau): & \inf _{\|\phi\|_{2}^{2}=(4 \pi \tau)^{\frac{n}{2}}} \mathcal{W}(g(x, t), \phi(x, t), \tau(t)) \\
& =\inf _{\|\phi\|_{2}^{2}=(4 \pi \tau)^{\frac{n}{2}}}(4 \pi \tau)^{-\frac{n}{2}} \int_{M}\left[\tau\left(4|\nabla \phi|^{2}+R \phi^{2}\right)-\phi^{2} \ln \phi^{2}-n \phi^{2}\right] d \operatorname{vol}_{g}
\end{aligned}
$$

is non-decreasing along the Ricci flow for any $\tau(t)$ and $\phi$ such that $\tau^{\prime}=-1$ and $\frac{\partial}{\partial t} \phi^{2}=-\Delta \phi^{2}+\left(R-\frac{n}{2 \tau}\right) \phi^{2}$.

Denote $V(t)=\operatorname{Vol}_{g(t)}, \tau_{0}=\tau(0), \tau=\tau(t)$, and consider $\phi^{2}=(4 \pi \tau)^{\frac{n}{2}} V(t)^{-1}$ at time $t$. So, by the monotonicity of $\mu$, one has

$$
\begin{aligned}
\mu\left(g(0), \tau_{0}\right) & \leq \mu(g(t), \tau) \\
& \leq(4 \pi \tau)^{-\frac{n}{2}} \int_{M}\left[\left(\tau R \phi^{2}-\phi^{2} \ln \phi^{2}-n \phi^{2}\right)\right] d \operatorname{vol}_{g(t)} \\
& =f_{M}[\tau R+\ln V(t)] d \operatorname{vol}_{g(t)}-\ln (4 \pi \tau)^{\frac{n}{2}}-n \\
& =-\tau \frac{d}{d t}(\ln V(t))+\ln V(t)-\ln (4 \pi \tau)^{\frac{n}{2}}-n
\end{aligned}
$$

and thus

$$
-\frac{d}{d t}(\tau \ln V(t)) \geq \frac{n}{2} \ln \tau+\frac{n}{2} \ln (4 \pi)+n+\mu\left(g(0), \tau_{0}\right) .
$$

Taking $\tau=T-t$ and integrating the inequality from $t$ to $T$, we obtain

$$
V(t) \geq(4 \pi)^{\frac{n}{2}} e^{\mu(g(0), T)+\frac{n}{2}}(T-t)^{\frac{n}{2}} .
$$


One may compare the upper bound of $\mu$ with a former result given by one of the authors as follows.

Proposition 3 ([ZZha07], cf. [CCG+10]). For any closed Riemannian manifold $\left(M^{n}, g\right)$, one has

$$
\mu(g, T) \leq-n+T \lambda+e^{-1} \operatorname{Vol}_{g}(M)-\frac{n}{2} \ln 4 \pi T .
$$

Moreover, if $\lambda \leq 0$, then

$$
\mu(g, T) \leq-n+e^{-1}+\ln \operatorname{Vol}_{g}(M)-\frac{n}{2} \ln 4 \pi T .
$$

For the reader's convenience, we recall the proof.

Proof. The first inequality comes from the definition of $\mu$ and the fact $-x \ln x \leq e^{-1}$ for all $x \geq 0$. When $\lambda<0$, we can simply remove the term $T \lambda$. However, a rescaling argument can do a better job. Indeed, since $\mu(g, T)=\mu(Q g, Q T)$ for any $Q \in \mathbb{R}$, when choosing $Q=\operatorname{Vol}_{g}^{-\frac{2}{n}}$, one has $\operatorname{Vol}_{Q g}(M)=1$,

$$
\mu(Q g, Q T) \leq-n+Q T \lambda+e^{-1} \operatorname{Vol}_{Q g}(M)-\frac{n}{2} \ln 4 \pi Q T \leq-n+e^{-1}-\frac{n}{2} \ln 4 \pi Q T
$$

and thus the proposition is proved.

More discussions about the behavior of $\mu$ and its applications can be found in CCG+10, Chapter 17].

\section{UPPER BOUNDS}

Let $g(t), t \in[0, T)$, be the solution of the Ricci flow on a closed Riemannian manifold $\left(M^{n}, g(0)\right)$. Along this flow, consider the heat kernel $G(x, t ; y, s)$ for the heat operator $\partial_{t}-\Delta_{x}$. Namely, fixing $y$ and $s, u(x, t):=G(x, t ; y, s)$ satisfies

$$
\partial_{t} u(x, t)=\Delta_{x} u(x, t) \text { and } \lim _{t \searrow_{s}} u(x, t)=\delta_{y}(x) .
$$

One can consult Chow et al.'s book [CCG+10, Ch. 24] for more details about the heat kernel. In QZha12, pp. 247, 251], Q. Zhang derived the following two-sided bound for the integral heat kernel:

$$
\begin{aligned}
(1+C(t-s))^{\frac{n}{2}} & \geq \int_{M} G(x, t ; y, s) d \mu_{g(s)}(x) \\
& \geq \frac{C}{(t-s)^{\frac{n}{2}}} \exp \left(-C \frac{\operatorname{dist}_{g(t)}^{2}(x, y)}{t-s}-\frac{1}{2 \sqrt{t-s}} \int_{s}^{t} \sqrt{t-\tau} R\left(x_{0}, \sigma\right) d \sigma\right),
\end{aligned}
$$

where $C$ 's are constants depending on $n, T$ and $g(0)$. Hence, by our assumptions on the scalar curvature and the diameter, one obtains the following theorem, which is essentially due to Q. Zhang in QZha12.

Proposition 2 (cf. Theorem 1.1 (a) in QZha12]). Let $\{g(t)\}_{t \in[0, T)}$ be the solution of the Ricci flow on a closed Riemannian manifold $\left(M^{n}, g(0)\right)$ with $n \geq 3$. If the diameter satisfies $\operatorname{diam}_{g(t)} \leq c_{1} \sqrt{T-t}$ and there exist a point $x_{0} \in M$ such that $R\left(x_{0}, t\right) \leq$ $c_{2}(T-t)^{-1}$, then we have $\operatorname{Vol}_{g(t)} \leq C(T-t)^{\frac{n}{2}}$ for all $t>\frac{T}{2}$, where $C$ depends only on $c_{1}, c_{2}, n, T$ and $g(0)$. 
Proof. The proof is adapted from Zhang's local volume estimate in QZha12. The reader should be careful on tracing the dependence of the constant $C$, which varies line by line, in the following bounds.

Recall that $R(x, t)$ is either nonnegative, or negative somewhere and bounded below by the negative function $\left(\frac{1}{\min R_{g(0)}}-\frac{2 t}{n}\right)^{-1}$ for all $t>0$. Moreover, since the manifold is closed and $\frac{d}{d t} d \mu_{g(t)}=-R d \mu_{g(t)}$, one can derive

$$
\frac{d}{d t} \int_{M} u(x, t) d \mu_{g(t)}=\int_{M} \Delta_{x} u(x, t)-R u(x, t) d \mu_{g(t)}=-\int_{M} R u(x, t) d \mu_{g(t)}
$$

and thus either $\frac{d}{d t} \int_{M} u(x, t) d \mu_{g(t)} \leq 0$ or

$$
\frac{d}{d t} \int_{M} u(x, t) d \mu_{g(t)} \leq \frac{n}{2}\left(t-\frac{n}{2 \min R_{g(0)}}\right)^{-1} \int_{M} u(x, t) d \mu_{g(t)} .
$$

Integrating it from $s$ to $t$, we obtain either

or

$$
\int_{M} u(x, t) d \mu_{g(t)} \leq \lim _{t \searrow s} \int_{M} u(x, t) d \mu_{g(t)}=1
$$

$$
\int_{M} u(x, t) d \mu_{g(t)} \leq\left(\frac{t-\frac{n}{2 \min R_{g(0)}}}{s-\frac{n}{2 \min R_{g(0)}}}\right)^{\frac{n}{2}} \leq\left(1+C_{1}(t-s)\right)^{\frac{n}{2}},
$$

where $C_{1}=\left(-\frac{n}{2 \min R_{g(0)}}\right)^{-1}$. Thus the upper bound for the integral heat kernel is obtained.

We claim that $G\left(x, t ; x_{0}, s\right)$ is bounded pointwise from below by $C_{2}(t-s)^{-\frac{n}{2}}$ for all $t \geq \frac{T+s}{2}$, where $C_{2}$ depends on $c_{1}, c_{2}, n, T$ and $g(0)$. Therefore, combining with the upper bound above, we have

$$
\left(1+C_{1}(t-s)\right)^{\frac{n}{2}} \geq \int_{M} G\left(x, t ; x_{0}, s\right) d \mu_{g(t)}(x) \geq C_{2}(t-s)^{-\frac{n}{2}} \operatorname{Vol}_{g(t)}
$$

and thus

$$
\mathrm{Vol}_{g(t)} \leq C\left((t-s)+(t-s)^{2}\right)^{\frac{n}{2}} \leq C(t-s)^{\frac{n}{2}}
$$

where the last $C$ depends only on $n, C_{1}, C_{2}$ and $T$. This upper bound holds for all fixed $s$ and all $t \in\left[\frac{T+s}{2}, T\right]$, so we may choose $t=\frac{T+s}{2}$ and derive the conclusion

$$
\operatorname{Vol}_{g(t)} \leq C(T-t)^{\frac{n}{2}} \text { for all } t>T / 2 .
$$

Now we complete the proof by verifying the claim that $G\left(x, t ; x_{0}, s\right) \geq C_{2}(t-s)^{-\frac{n}{2}}$. Note that, fixing $x$ and $t, v(y, s):=G(x, t ; y, s)$ satisfies the backward conjugate heat equation $\partial_{s} v=-\Delta_{y} v+R v$ and thus the function $f(y, s)$ defined by $(4 \pi \tau)^{-\frac{n}{2}} e^{-f}=v$ satisfies $-f_{s}=\Delta f-|\nabla f|^{2}+R-\frac{n}{2 \tau}$, where $\tau=t-s$. Combining with Perelman's estimate $\tau\left(2 \Delta f-|\nabla f|^{2}+R\right)+f-n \leq 0$ (cf. [Per02, Corollary 9.4]), one has

$$
-f_{s} \leq \frac{1}{2} R-\frac{1}{2}|\nabla f|^{2}-\frac{1}{2 \tau} f \leq \frac{1}{2} R-\frac{1}{2 \tau} f, \quad \text { i.e., } \quad-(\sqrt{\tau} f)_{s} \leq \frac{1}{2} \sqrt{\tau} R .
$$

Integrating from $s$ to $t$, one has

$$
\sqrt{t-s} f(y, s) \leq \lim _{\sigma \rightarrow t} \sqrt{t-\sigma} f(y, \sigma)+\frac{1}{2} \int_{s}^{t} \sqrt{t-\sigma} R(y, \sigma) d \sigma .
$$


Because $G(x, t ; y, \sigma)$ behaves like $(t-\sigma)^{-\frac{n}{2}}$ as $\sigma \rightarrow t$ whenever $x=y$ (cf. [CCG+10, Ch. 24]), $f(x, \sigma)$ is uniformly bounded as $\sigma \rightarrow t$, thus $\lim _{\sigma \rightarrow t} \sqrt{t-\sigma} f(x, \sigma)=0$ and

$$
\sqrt{t-s} f(x, s) \leq \frac{1}{2} \int_{s}^{t} \sqrt{t-\sigma} R(x, \sigma) d \sigma .
$$

Since $x$ can be chosen arbitrarily, one may take $x=x_{0}$ in the beginning and obtain

$$
-f\left(x_{0}, s\right) \geq-\frac{1}{2 \sqrt{t-s}} \int_{s}^{t} \sqrt{t-\sigma} R\left(x_{0}, \sigma\right) d \sigma \geq-c_{2} .
$$

So

$$
G\left(x_{0}, t ; x_{0}, s\right)=v\left(x_{0}, s\right) \geq(4 \pi(t-s))^{-\frac{n}{2}} e^{-c_{2}} .
$$

Moreover, by gradient estimate of heat equation along the Ricci flow (cf. QZha06, $(3.44)])$, one can compare $v(x, s)$ with $v\left(x_{0}, s\right)$, i.e.,

$$
G\left(x, t ; x_{0}, s\right) \geq C_{3} K^{-1} \exp \left(-2 C_{4} \frac{\operatorname{dist}_{g(t)}^{2}\left(x, x_{0}\right)}{t-s}\right)\left(G\left(x_{0}, t ; x_{0}, s\right)\right)^{2}
$$

for all $x \in M$, where $K$ is the upper bound of $G$ and $C_{3}, C_{4}$ are universal constants. In QZha12, (1.5)], it was proved that $K \leq C_{5}(t-s)^{-\frac{n}{2}}$. Therefore, using $\operatorname{diam}_{g(t)} \leq$ $c_{1} \sqrt{T-t}$ and $t \geq \frac{T+s}{2}$, one has

$$
\begin{aligned}
G\left(x, t ; x_{0}, s\right) & \geq C_{3} K^{-1} \exp \left(-2 C_{4} \frac{\operatorname{dist}_{g(t)}^{2}\left(x, x_{0}\right)}{t-s}\right)\left(G\left(x_{0}, t ; x_{0}, s\right)\right)^{2} \\
& \geq C_{3}(4 \pi)^{-n} e^{-2 c_{2}} C_{5}^{-1} \exp \left(-2 C_{4} \frac{2 c_{1}^{2}(T-t)}{T-s}\right)(t-s)^{-\frac{n}{2}} \\
& \geq C_{2}(t-s)^{-\frac{n}{2}}, \quad \text { where } C_{2}=(4 \pi)^{-n} C_{3} C_{5}^{-1} e^{-2 c_{2}-4 C_{4} c_{1}^{2}} .
\end{aligned}
$$

The claim is verified for $C_{2}$ depending only on $c_{1}, c_{2}, n, T$ and $g(0)$.

Remark 1. Suppose that $f R(x, t) d \operatorname{vol}_{g(t)} \geq \frac{n}{2}(T-t)^{-1}$ for all $t>\frac{T}{2}$. Then

$$
\frac{d}{d t} \operatorname{Vol}_{g(t)}=-\int_{M} R d \operatorname{vol}_{g(t)} \leq-\frac{n}{2}(T-t)^{-1} \operatorname{Vol}_{g(t)}
$$

implies that $\operatorname{Vol}_{g(t)} \leq C(T-t)^{\frac{n}{2}}$ for all $t>\frac{T}{2}$, where $C=\left(\frac{2}{T}\right)^{\frac{n}{2}} \operatorname{Vol}_{g\left(\frac{T}{2}\right)}$. This might help to remove the curvature assumption in Theorem 2. We remind the reader that, when $f R(x, t) d \operatorname{vol}_{g(t)} \nsupseteq \frac{n}{2}(T-t)^{-1}$ for some $t_{k} \rightarrow T$, there must exist a sequence of points $\left(x_{k}, t_{k}\right)$ with Type I blow-up scalar curvature. However, this is insufficient for us to apply Theorem 2 because we need a fixed point $x_{0}$.

Remark 2. For generic Ricci flows, it is not hard to see that volume grows at most polynomially. Indeed, this is trivial when $R_{g(0)} \geq 0$. For $\min R_{g(0)}<0$, by using $\frac{d}{d t} R \geq \Delta R+\frac{2}{n} R^{2}$, one can show that $R_{g(t)} \geq\left(\frac{1}{\min R_{g(0)}}-\frac{2 t}{n}\right)^{-1}$ and thus $\frac{d}{d t} \ln \operatorname{Vol}_{g(t)}=$ $-f_{M} R d \operatorname{vol}_{g(t)} \leq\left(\frac{2 t}{n}-\frac{1}{\min R_{g(0)}}\right)^{-1}$. Hence

$$
\operatorname{Vol}_{g(t)} \leq \operatorname{Vol}_{g(0)}\left(-\min R_{g(0)}\right)^{\frac{n}{2}}\left(\frac{2 t}{n}-\frac{1}{\min R_{g(0)}}\right)^{\frac{n}{2}}
$$

for all $t<T$. 


\section{REFERENCES}

[Aub76] T. Aubin. Équations différentielles non linéaireset problème de Yamabe concernant la courbure scalaire. J. Math. Pures Appl., 55:269-296, 1976.

$[\mathrm{CCG}+10]$ B. Chow, S.-C. Chu, D. Glickenstein, C. Guenther, J. Isenberg, T. Ivey, D. Knopf, P. Lu, F. Luo, and L. Ni. The Ricci flow: Techniques and Applications. Part III: GeometricAnalytic Aspects, volume 163 of Math. Surv. Mono. Amer. Math. Soc., 2010.

[DH02] O. Druet and E. Hebey. The $A B$ program in geometric analysis: sharp Sobolev inequalities and related problems. Mem. Amer. Math. Soc., 160(761), 2002.

[Hsu07] S.-Y. Hsu. Uniqueness of solutions of Ricci flow on complete noncompact manifolds. arXiv:0704.3468v4, 2007.

[HV96] E. Hebey and M. Vaugon. Meilleures constantes dans le théorèm dinclusion de Sobolev. Ann. Inst. H. Poincaré (C) Anal. Non Linéaire, 13:57-93, 1996.

[Per02] G. Perelman. The entropy formula for the Ricci flow and its geometric applications. arXiv:math/0211159v1, 2002.

[Ye07] R. Ye. The logarithmic Sobolev inequality along the Ricci flow. arXiv:0707.2424v4, 2007.

[QZha06] Q. S. Zhang. Some gradient estimates for the heat equation on domains and for an equation by Perelman. Int. Math. Res. Note, pages 1-39, Art. ID 92314, 2006.

[QZha07] Q. S. Zhang. A uniform Sobolev inequality under Ricci flow. arXiv:0706.159404, 2007.

[QZha12] Q. S. Zhang. Bounds on volume growth of geodesic balls under Ricci flow. Math. Res. Lett., 19(1):245-253, 2012.

[ZZha07] Z. Zhang. Compact blow-up limits of finite time singularities of Ricci flow are shrinking Ricci solitons. C. R. Math. Acad. Sci. Paris, 345(9):503-506, 2007.

(Chih-Wei Chen) National Kaohsiung Normal University, Taiwan

E-mail address: BabbageTW@gmail.com; chencw@nknu.edu.tw

(Zhenlei Zhang) Department of Mathematics, Capital Normal University, Beijing, CHINA

E-mail address: Zhleigo@aliyun.com 